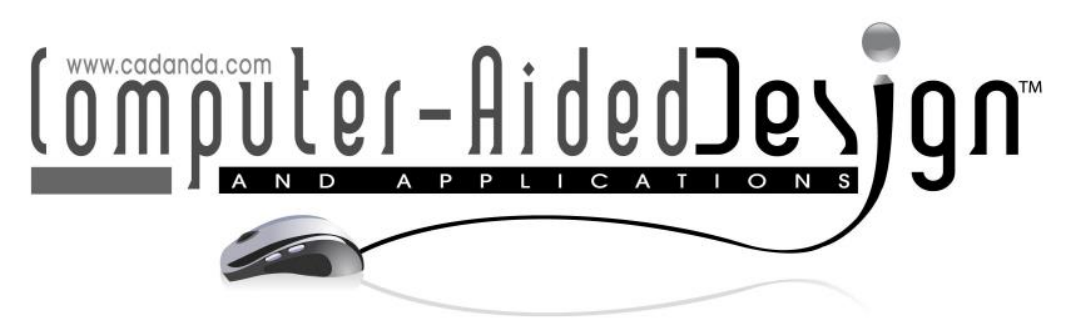

\title{
3D CAD Information System Integration Based on the Private Cloud Platform
}

\author{
Yujie Sun'(iD) \\ ${ }^{1}$ Technology \& Media University of Henan Kaifeng, KaiFeng, Henan 475004, China, \\ kcyjs2021@126.com
}

Corresponding author: Yujie Sun, kcyjs2021@126.com

\begin{abstract}
With the rapid development of a new generation of information technology such as cloud computing, 3D CAD technology is becoming the platform foundation for product innovation design and digital design and manufacturing of manufacturing enterprises. This paper proposes research on the 3D CAD information systems integration based on a private cloud platform. Through studying the concept, characteristics and hierarchical structure of cloud computing. According to the manufacturing information application requirements, the system will publish and deploy corresponding application services according to user needs, and effectively solve the poor integration of some current information systems. Private cloud service platform. In view of the wide variety of 3D CAD software currently on the market, in the process of integrating with the manufacturing information system, a general integration framework design based on the cloud platform is proposed and standardized cloud service interface definitions are provided to extend the functions of the integration framework. Data differences between different CADs, and XML files with good scalability and data self-describing capabilities are used as intermediate files for information interaction. Through the entire business process, the technical application of engineering drawing into PDF, browsing and signing was introduced, and the correctness of the private cloud platform and integration technology was verified.
\end{abstract}

Keywords: Computer technology; supply chain finance; risk control; financial technology

DOI: https://doi.org/10.14733/cadaps.2022.S6.1-12

\section{INTRODUCTION}

At present, the development of my country's manufacturing industry is constantly transforming from a low-end processing industry to a high-end service industry. The upgrading of manufacturing with high added value has become a new growth point supporting economic development. Gupta et al. [1] believed that the realization of this type of business transformation 
requires the support of platforms, new technologies and new models. In recent years, a servicebased computing model-cloud has flourished, and Arvor et al. [2] believed the concept of "everything is a service" has emerged, which makes computing resources a professional service and is provided through information.

Although software systems such as CAX, CAPP, PDM and MES are very good at solving user needs in their respective fields. However, in the face of collaborative applications between systems, the current methods are not very good. Most of them are at the cost of a large amount of information redundancy. That is, the same information is frequently imported and exported in different systems. Unknown, management data is too complicated, too much and it becomes slower and slower. In the face of complex system engineering, it is necessary to comprehensively utilize the information of the existing information system to realize the safe use and sharing of information. Due to the fear of being replaced, the degree and depth of cooperation among various software vendors are not ideal. With the acceleration of the restructuring and optimization of manufacturing enterprises, the business of the enterprise presents a trend of group management: decentralized design and manufacturing, centralized procurement and management. Tran et al. [3] believed that solving the problems of remote application in foreign offices, remote application of outsourcing processing manufacturers, remote process signing of branch plants, and remote information release of workshops have brought huge challenges to traditional information management methods.

Due to the emergence of Internet of Things and network cloud computing services, the development of the information industry has found a new fulcrum. Internet of Things technology and cloud computing have been widely used in the public service industry. Foreign companies such as Amazon and Google have used these new technologies to upgrade the company's information system and have obtained huge benefits; domestic Internet companies such as Sina and Taobao. Khan et al. [4] believed that the new technologies are also injected, but this is limited to the service industry of the tertiary industry.

Therefore, this paper will draw lessons from cloud service technology combined with Kaimu's long-term experience in manufacturing information technology research and development and service. Aiming at the status quo of domestic enterprises, we will independently develop a private cloud service platform for China's manufacturing industry. Barladian et al. [5] believed that twodimensional CAPP, three-dimensional machine plus CAPP, three-dimensional assembly CAPP and other design and manufacturing software upgrades and cloud service deployment, reconstruct the information service system of manufacturing enterprises, and develop a "private cloud service platform for the full life cycle of manufacturing products."

\section{RELATED STUDIES}

\subsection{Research Status of Private Cloud Platform in Manufacturing Industry at Home and Abroad}

At present, the domestic manufacturing industry has widely used software systems such as CAX, PDM, CAPP, and MES. Although they can solve problems well in their respective fields of expertise, the cooperation between the software systems is not deep. Because each software system is in independent operations, Relvas and Ramos [6] believed that many of the same product information has to be imported and exported repeatedly, which brings a lot of redundant information. In addition, the current process information release systems in China can only release two-dimensional assembly information. However, some special assembly relationships cannot be fully expressed only by two-dimensional diagrams. If combined with three-dimensional diagrams, the assembly relationships can be expressed more intuitively in Figure 1. 


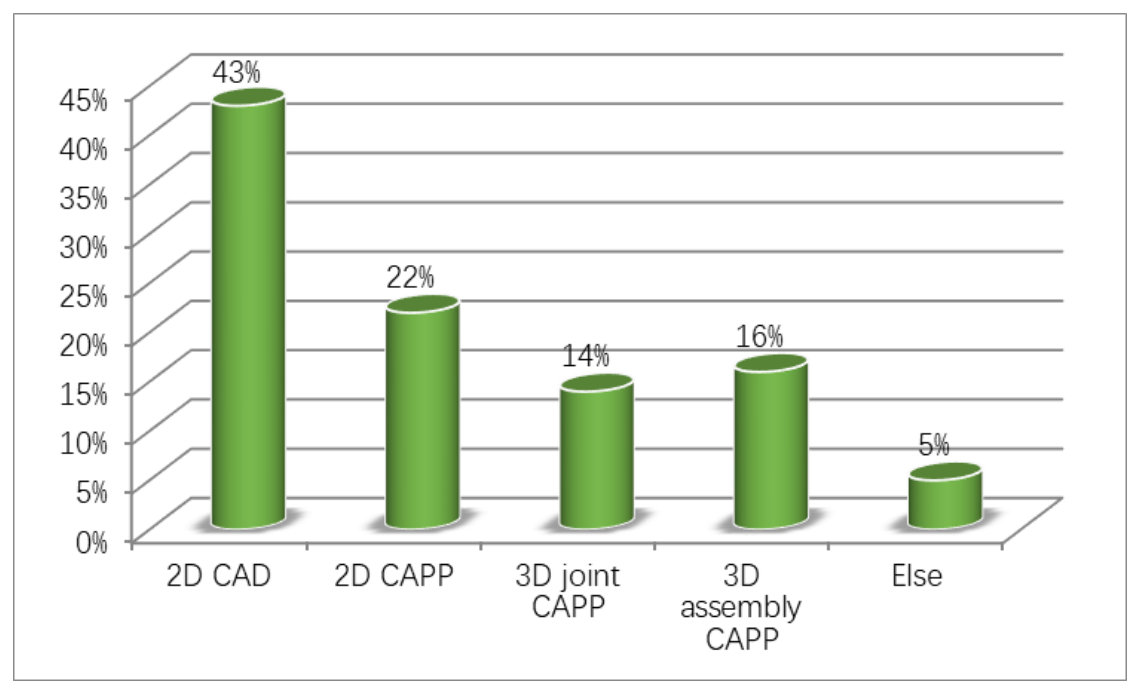

Figure 1: The proportion of computer-aided design technology in engineering design.

At present, foreign PLM manufacturers in the Chinese market mainly focus on product promotion in the process industry. In the discrete manufacturing industry, they are still in the stage of trying to enter. For example, foreign manufacturers Siemens rely on their own 3D design software to provide their own 3D assembly system, such as Siemens provides a simulation system for the entire assembly process of PLM solutions. In terms of machine tool management, Germany has already invested in machinery industry companies using cloud computing to manage parts. Cimsource Software Company has specially developed a solution based on Web services to simplify the tool management of machine tools. While improving its flexibility, it reduces costs. No manufacturer based on the existing system logic data as the core has been found in the domestic market to be able to fully use the commercialized 3D assembly shop information publishing system. In addition, some manufacturers, universities, and research institutes have conducted researchbased product development under state funding.

\subsection{The Current Situation of the Role of Computer Technology in the Financial Risk Control System of the Supply Chain}

We mainly study the 3D CAD integration on a private cloud platform and manufacturing information system. Wang and $\mathrm{Bi}$ [7] believed that information system software mainly includes PDM, CAPP, ERP and MES, but they all have integration requirements with 3D CAD in practice. It is briefly introduced the more 3D CAD software and research on its secondary development method.

CATIA is the flagship solution for product development of the French company Desalt Systems. Due to its strong hybrid modeling capabilities of curved surfaces and solids, it has been widely used in aerospace, automotive and shipbuilding industries. CATIA's secondary development methods mainly divided into two categories: one is the Automation method, and the other is the Component Application Architecture method. Automation technology is a COM-based technology, specifically developed from the continuous evolution of object link embedded automation technology. It is relatively easy to get started, but the developed software functions are more limited; while CAAC ++ is using rapid application development environment RADE (Rapid Application Development Environment) and different API application program interfaces to complete, can carry out from simple to complex and the most efficient application development. Cai and Wang [8] believed that RADE is a visual integrated development environment, integrated in Microsoft Visual $\mathrm{C}++$, adding CAA development tool set in the $\mathrm{VC}++$ environment in Figure 2. 


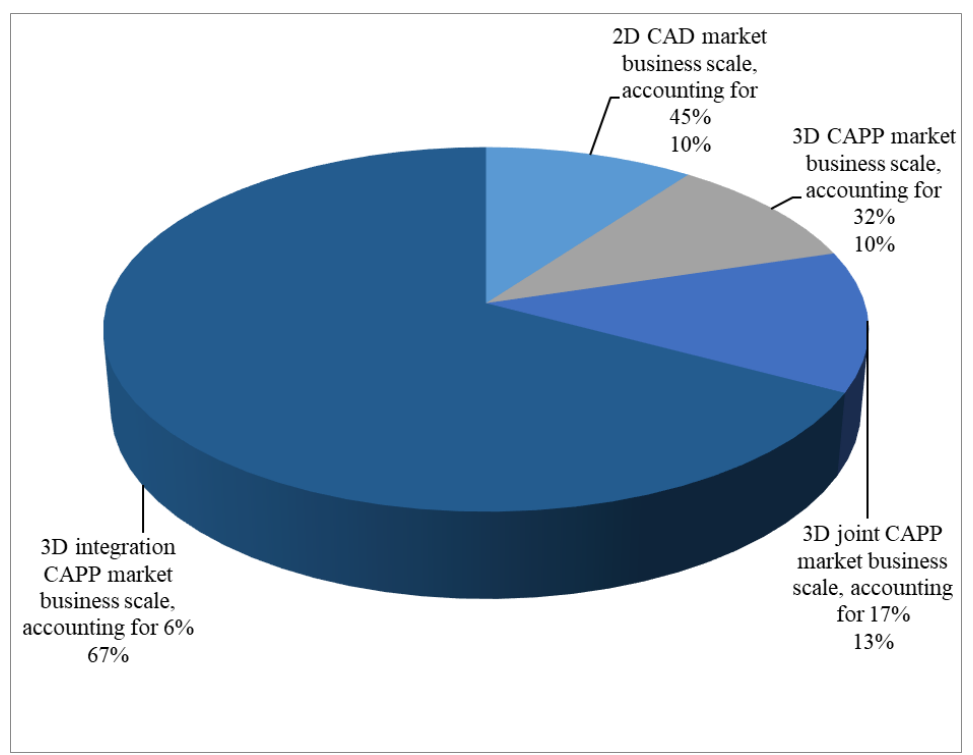

Figure 2: The business share of CAD and CAPP products in the market.

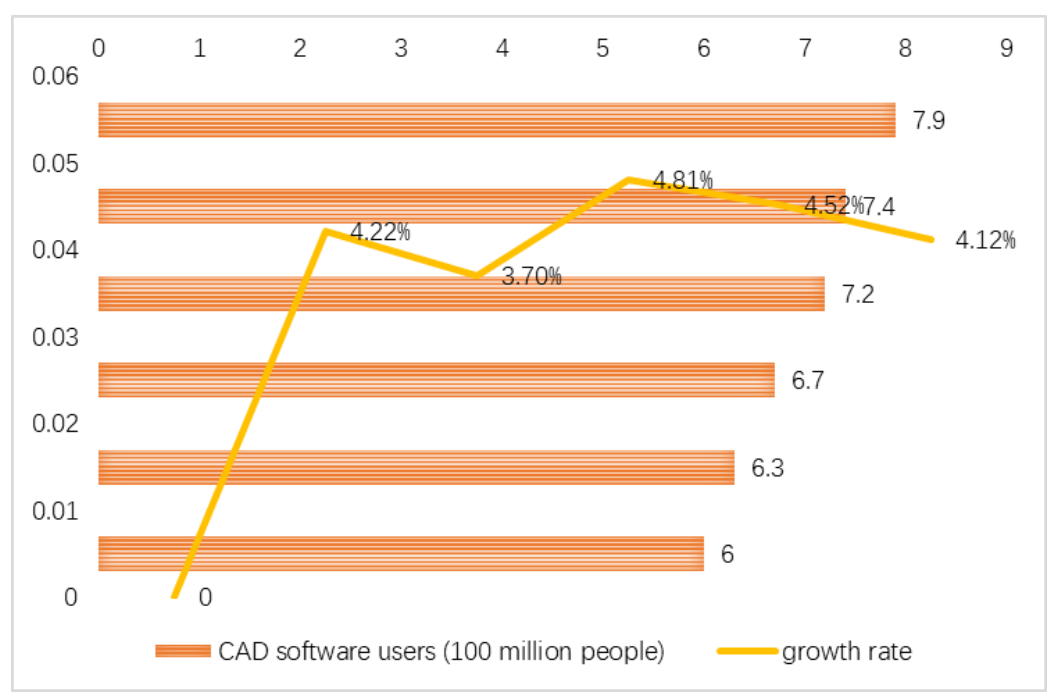

Figure 3: The number and growth rate of 3D-CAD software users from 2015 to 2020.

Uni-graphics NX is a product engineering solution produced by Siemens PLM Software. It has powerful functions and can easily realize the construction of various complex entities and shapes. It is widely used in aerospace, automotive, machinery, shipbuilding and other fields. Krystek et al. [9] believed that the secondary development methods of UG mainly include Open Grip, Open API, UI Style and Open C++. Among them, Open API, also called Open C, is the most powerful secondary development tool. Its applications can be divided into two modes, namely internal Program mode and external program mode. The executable file obtained after the external program compiled and connected an executable program independent of UG; the executable file obtained after the internal program compiled and connected can only run in the UG environment in Figure 3. 
Solid Works is the world's first 3D Windows CAD operating system. Its software has powerful functions and a wide range of components, but it is simple and convenient to operate, easy to learn and easy to use. Configuration management is a unique part of the Solid Works software architecture, which involves part design, assembly design and engineering drawings. Configuration management enables you to derive different parts or assemblies through the transformation and combination of different parameters in a CAD document. Mokni et al. [10] believed that the secondary development of Solid Works divided into two types. One is ID is patch technology based on OLE Automation. This technology can only develop programs in the form of EXE. The developed software cannot directly linked to the Solid Works system and cannot be integrated with Solid Works; the other this development method based on COM, and users can directly access Solid works through API for secondary development program design in Figure 4.

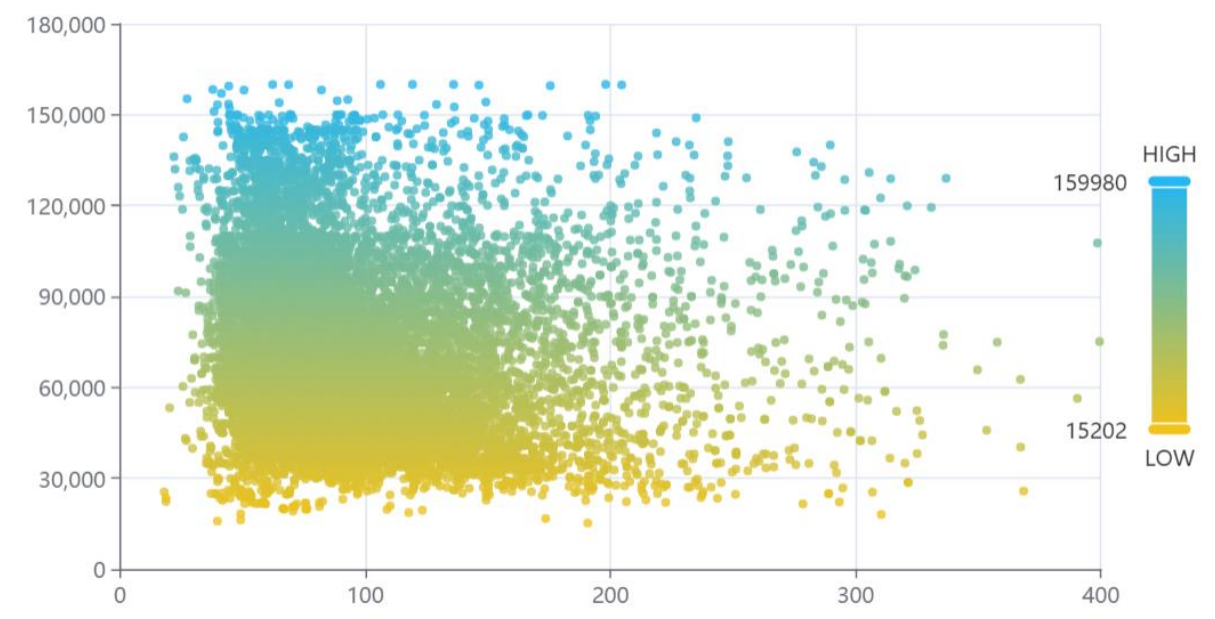

Figure 4: Relevance of 3D CAD system design and informatization penetration rate.

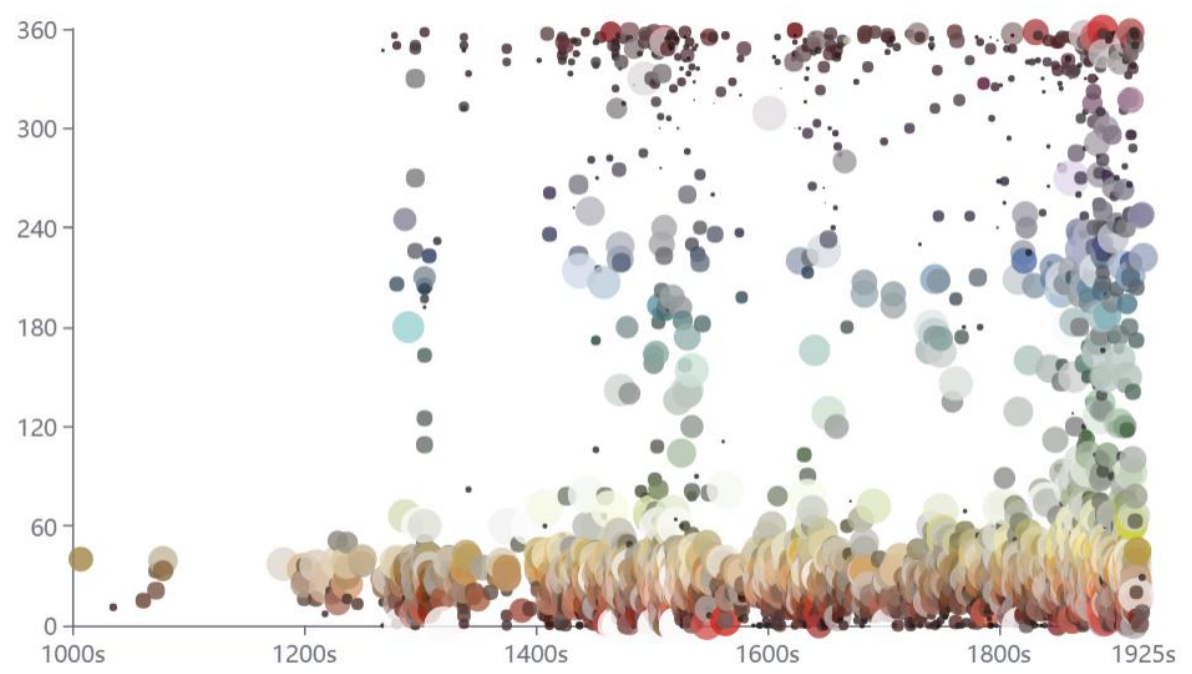

Figure 5: CAD software utilization rate and engineering design relevance.

Pro/Engineer is a 3D software integrated CAD/CAM/CAE under the American Parametric Technology Corporation (PTC). It is famous for parameterization and is the earliest application of 
parameterization technology. There are 5 ways of secondary development, namely Pro/TOOLKIT, Automation GATEWAY, J-Link, Pro/Web Link and VBAPI in Figure 5.

\section{ANALYSIS ON THE 3D CAD ASSEMBLY CAPP SYSTEM INTEGRATION}

\subsection{Integration of 3D CAD and 3D Assembly CAPP System}

There are close connections between different financial technology technologies. Liu Feng proposed "the future functions and structure of the Internet will be highly similar to the human brain, and it will also have Internet Virtual sensation, virtual movement, virtual center, virtual memory and nervous system" in "Internet Evolution" (published by Tsinghua University Press in September 2012). As companies pursue rapid product design and digitization of manufacturing, traditional CAPP systems based on two-dimensional CAD cannot meet corporate information manufacturing. The traditional manual-based assembly process design method cannot meet the highly integrated information requirements of the manufacturing industry. Therefore, the 3D auxiliary assembly process system design demand is constantly being raised. It makes the assembly process diagram image and intuitive, and can achieve explosive and the animation effect is used to refer to the assembly site operation, which is relatively intuitive and easy to understand. The threedimensional assembly CAPP system structure shown in Figure 6, which generally divided into three parts: three-dimensional model introduction, like assembly process and design planning output. 3D model introduction is to import the extracted model assembly information into the assembly process system and generate a simulation model. The assembly process planning mainly carries out the preliminary design of the assembly work, including the assembly strategy, implementation sequence, assembly process inspection, and assembly process visualization display. The output of design files is mainly to output three-dimensional assembly engineering files, and specifically guide on-site assembly operations through three-dimensional drawings.

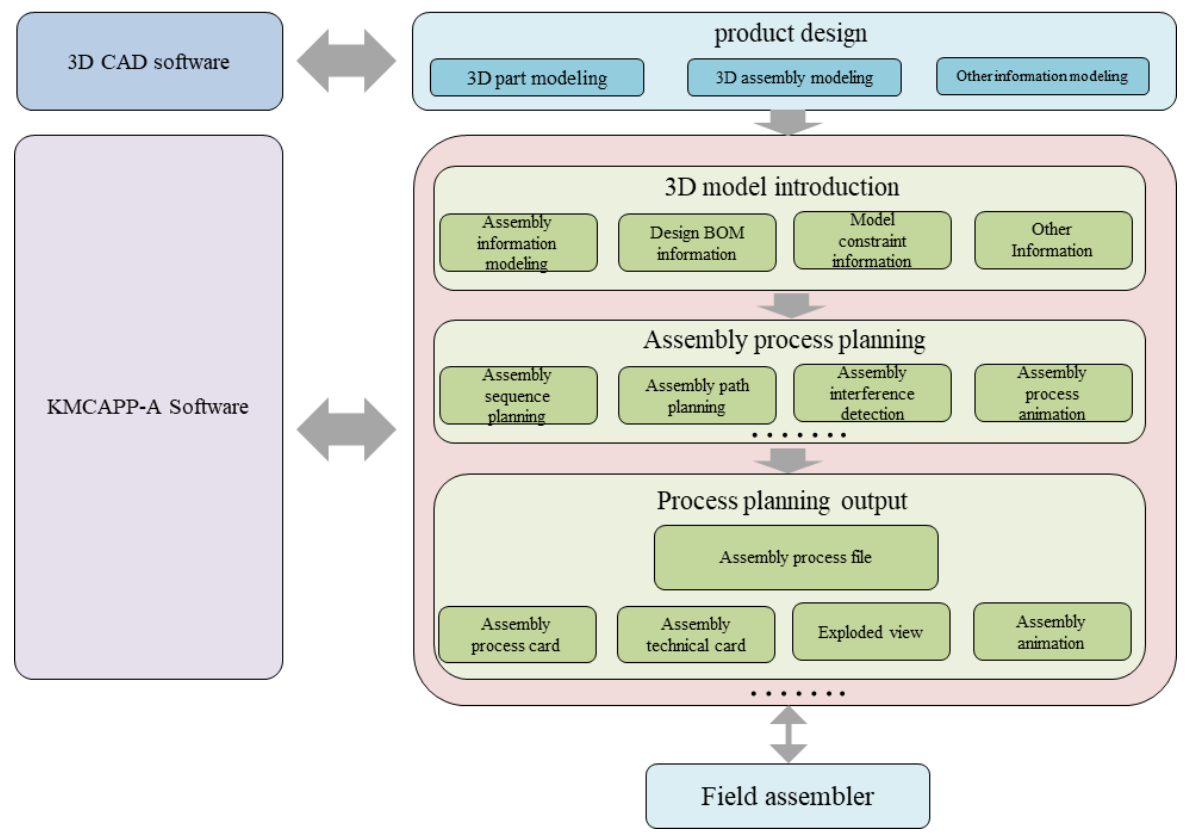

Figure 6: 3D CAD software assembly architecture.

In the integration process of 3D CAD software information system, a large amount of information data has been generated, such as document attribute information, model attribute information, 
assembly structure information, etc. It is extremely important as an intermediate file. This article uses expandable tags Language XML, which has many advantages such as platform portability, reusability, scalability, and self-explanation, and is a structured language in itself, which can clearly express various parent-child relationships such as component assembly and parameter tables. Therefore, XML files was used as the intermediate files for data exchange in the 3D CAD information systems integration. The XML process global information model shown in Figure 7.

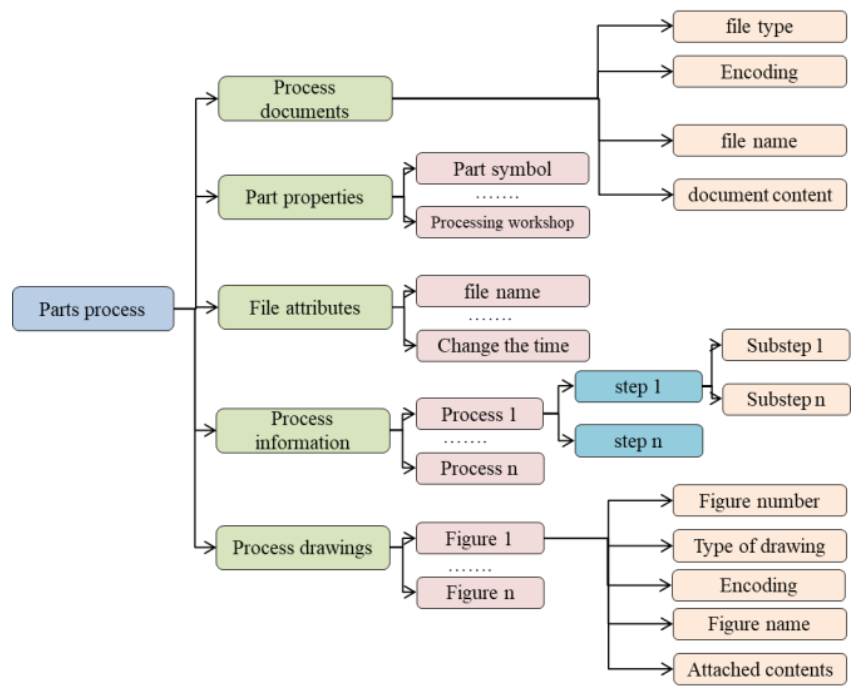

Figure 7: XML Process Global Information Model.

\subsection{Private Cloud Platform System and Integrated Architecture Design}

Cloud computing is an emerging shared infrastructure method that combines distributed, virtualized, and service-oriented (SOA) ideas to provide good dynamics and scalability for the infrastructure. The infrastructure of cloud computing-based information systems can be roughly divided into 3 layers, as shown in Figure 8. The bottom basic framework layer is to provide hardware resources required for software resource storage and operation, and play a supporting role in the system architecture. The virtual resource architecture layer is the core component level of cloud computing 3D-CAD applications. The service-oriented expansion of the underlying hardware resources through virtualization technology avoids the problems of fixed physical space distribution and storage space limitations, thereby realizing the regional sharing of computing resources. The emergence of cloud computing services has effectively improved computer computing and storage capabilities and expanded the effective coverage of resource services. Cloud computing providers specifically deploy and publish application services on the cloud user architecture level, and provide services to users through service nodes at different levels.

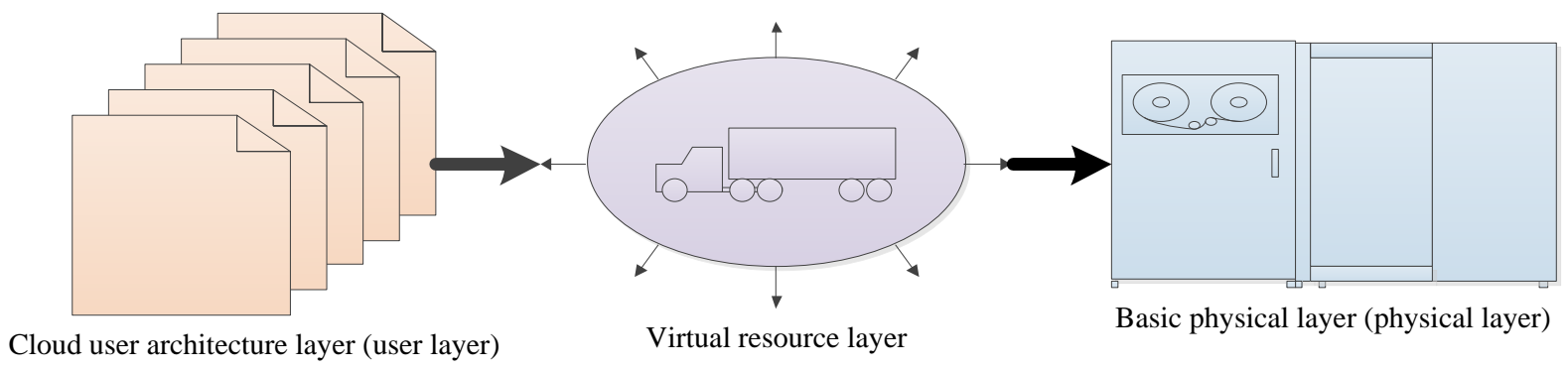

Figure 8: Cloud computing system infrastructure diagram. 
This paper proposes a 3D-CAD/CAPP service architecture for private cloud platforms, which continues the three-tier infrastructure concept commonly used by large Internet companies to build private cloud service systems, and realizes the distribution of information resources on the cloud platform architecture Style storage and release. The overall architecture deployment of the system is shown in Figure 9. In the three-tier cloud computing architecture system, the system architecture layer is mainly built on the cloud basic resource platform to achieve an effective connection between computing resource hardware infrastructure and user computing service resources. This research focuses on the cloud user architecture level. Within the system architecture level, the functional system is further subdivided into the cloud service scheduling level and the customer access level.

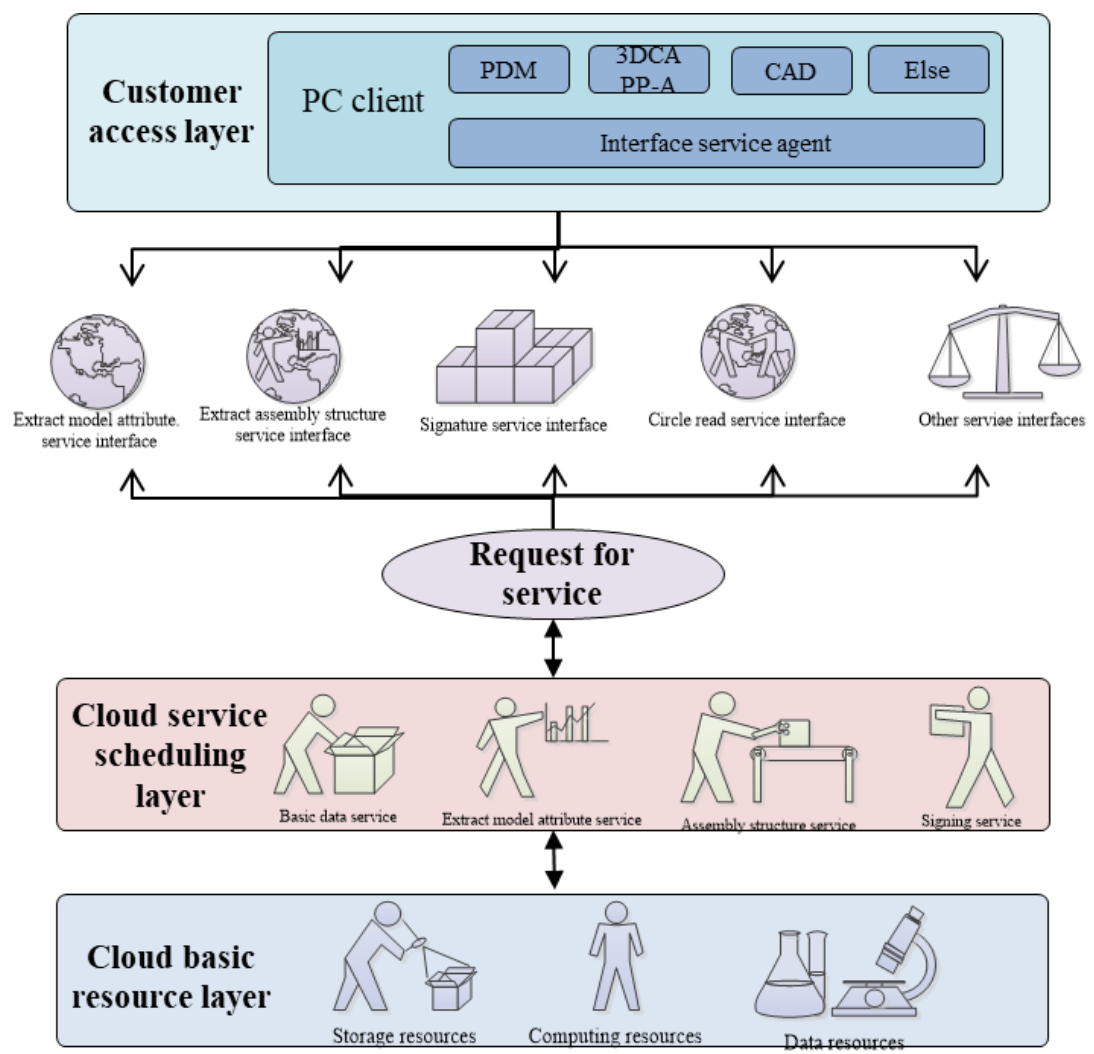

Figure 9: Overall architecture diagram of private cloud system.

\section{THE INTEGRATION FRAMEWORK OF 3D-CAD INFORMATION SYSTEM}

\subsection{D CAD Data Exchange Technology}

From the point of view of the data model that needs to be constructed for PDM integration, it mainly includes the structural relationship between the models, the composition of sub-building members, the assembly relationship of the system construction, the attributes of the detailed construction, and the engineering construction drawings. Therefore, it is necessary to adopt an object-oriented modeling method to construct a PDM model. The corresponding relationship between the data model of the PDM system and the 3D-CAD includes three parts. First, the PDM data model adopts the structure of building objects to express the organizational relationship 
between elements. Secondly, the feature aggregation relationship is used to express the assembly structure of the structural elements in the system, and the specific relationship feature attributes are stored in the part element object. Finally, in the PDM model, the descriptive documents are associated with the part elements in a one-to-one correspondence in Figure 10.

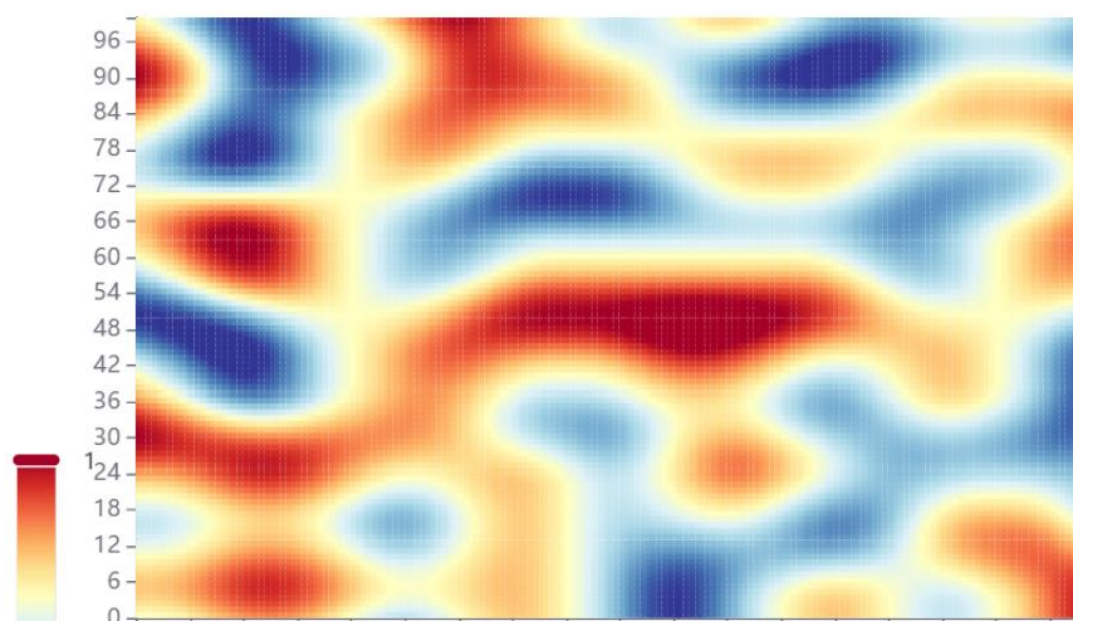

Figure 10: The application development distribution of CAD software system.

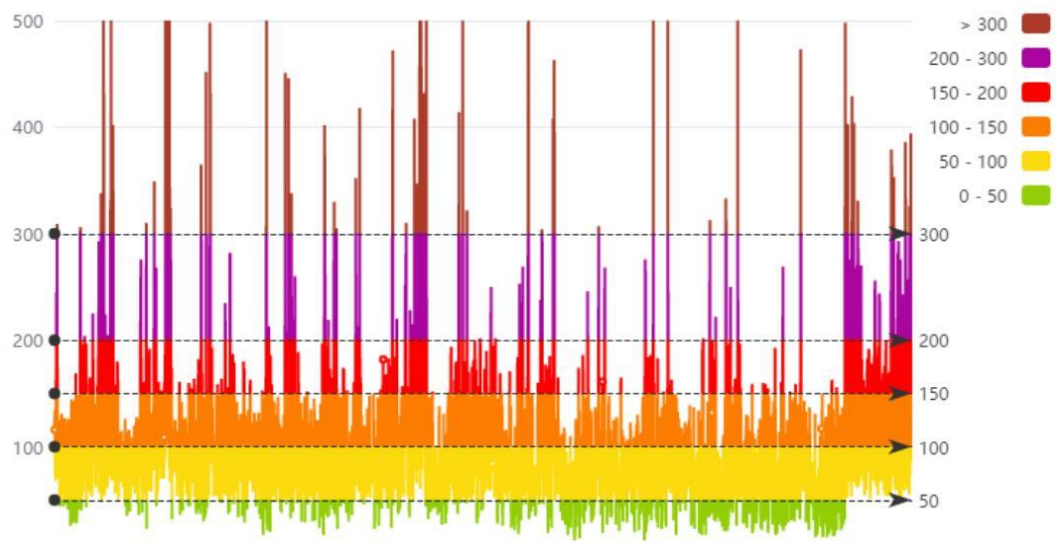

Figure 11: The number and growth rate of CAD software users from 2006 to 2020.

After sorting out the correspondence between the 3D-CAD system and the PDM data model, this article refines the data model conversion between the two software. Considering the API interface, forms of different 3D-CADs are different, and the data structure is also very different, the establishment of a unified data conversion model is the key content of the system connection. On the one hand, XML structure has many advantages such as portability, openness, platform independence and scalability, which is very suitable for expressing vectored content in CAD drawings in Figure 11. On the other hand, XML as a structured language can effectively express the relationship between the characteristic attributes and structural organization of different parts. On this basis, the DOM language is further used to decompose the XML file into object models, as to realize the data model conversion and connection between PDM and 3D-CAD in Figure 12. 


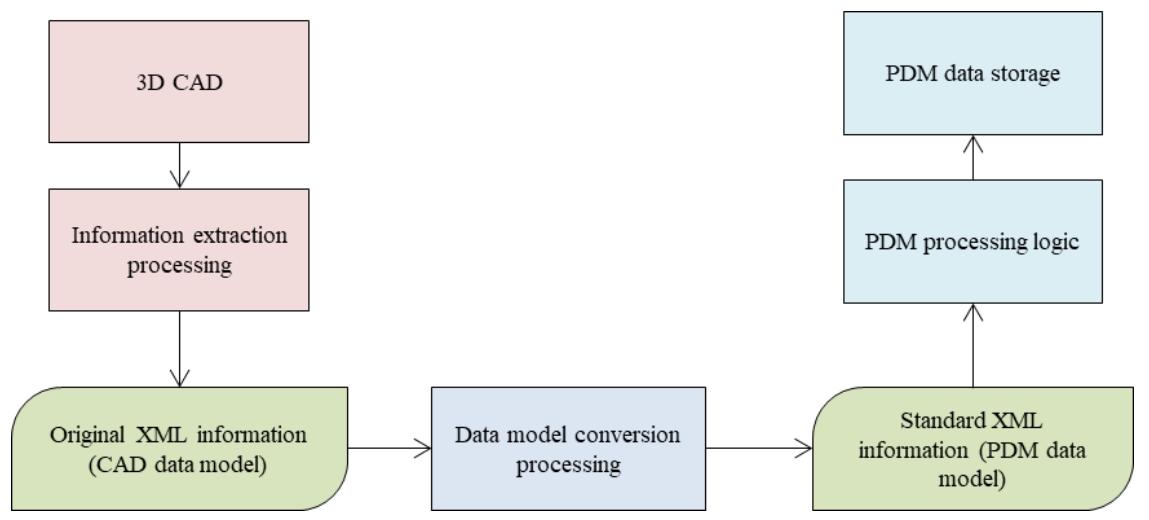

Figure 12: 3D CAD to PDM data conversion.

\subsection{D CAD Integrated Frame Structure}

The integration framework uses the component scheduler to uniformly schedule specific interfaces. There are three types of interfaces from 3D-CAD to PDM: The first is the universal 3D-CAD integrated interface. Since different 3D-CAD software has matching integrated components, it is necessary to extract the common features during system integration and store them in XML files to form a reusable CAD model. The result of the interface processing is shown as an XML file covering the CAD data model. The second is a data model conversion interface, through which the original CAD model performs data format conversion, and finally forms a standardized XML file. The last is the logical processing interface based on the PDM data model. This interface encapsulates the general processing logic of 3D data in the PDM model. The following Figure 13 shows the integrated structure block diagram of PDM and 3D-CAD. After the user has registered the relevant 3D-CAD integrated components, the PDM system calls the registered components through the component scheduler, and sends an edit or browse request to the CAD.

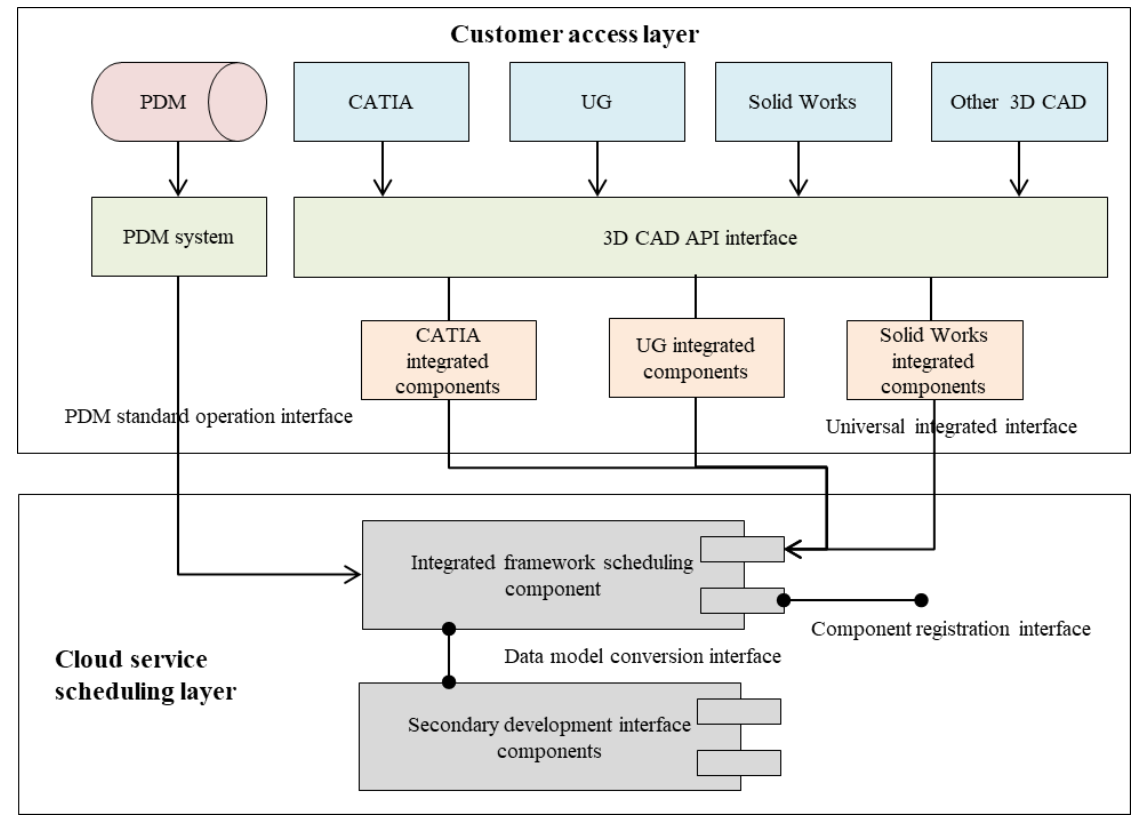

Figure 13: PDM and 3D CAD integrated frame structure. 


\subsection{D CAD/ CAPP Assembly Integration Framework}

Assembly structure information mainly includes component system information, component attribute information, and component hierarchical structure information. System information mainly includes the name, code, design time, design version, designer, technical standards, technical specifications, etc. of products and parts. Component attribute information mainly includes some physical information, such as quality, material, volume, density, color, etc. The component level includes the parent-child relationship between components, and the number of components defines the number of the same components in the same level. Component system information and component attribute information are the information of a single component. For assembly structure information, the difficulty is to study the extraction of component hierarchical structure information. The general assembly model structure hierarchy is shown in the following Figure 14:

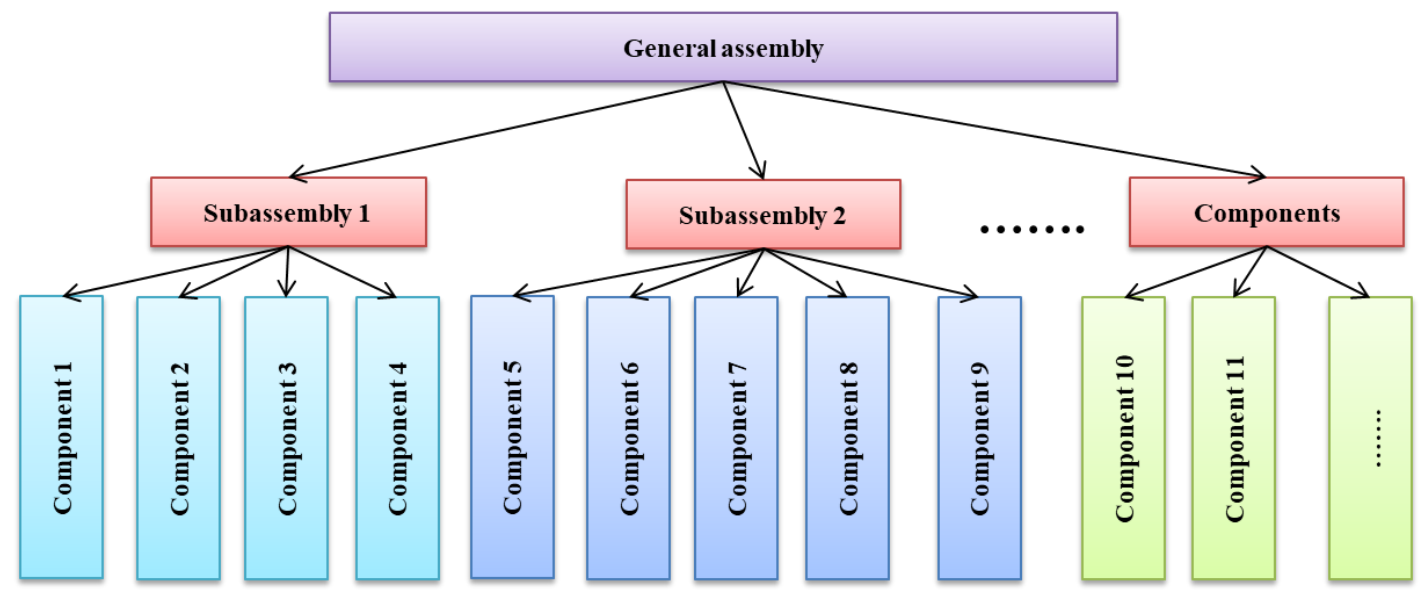

Figure 14: Assembly model structure hierarchy.

\section{CONCLUSION}

This paper studies the cloud platform construction of manufacturing information software with a private cloud service platform as the core. It mainly uses the PDM and 3D assembly CAPP of Kaimu Information Software Company as the platform, and introduces a variety of 3D CAD software in information extraction and document conversion. The related technologies of integration and attribute editing, specifically including the following five aspects: 1) Propose a private cloud service platform architecture for manufacturing enterprises based on cloud computing and Internet of Things and other related technologies, and turn local applications into cloud services. Realize different application requirements through the services provided by the cloud service scheduling layer. 2) For the integration of 3D CAD, a general integration framework design based on a private cloud platform is proposed. It shields the data differences between CAD and uses a unified data format to exchange data with PDM and 3D assembly CAPP.

In other words, the use of XML format intermediate files effectively solves the effective transmission of unstructured data, and the structured data form itself can clearly express the parent-child relationship between the assembly of parts and parameter tables. 3) Taking CATIA as an example, it focuses on the assembly structure information service. Using the secondary development method of CAA $\mathrm{C}++$, the method of extracting assembly structure information and model file attribute information based on the assembly model file is proposed, and the pipeline model is used as the example specifically introduces the extraction method of its geometric information. 4) Taking SolidWorks as an example, it focuses on the part family information service, mainly based on the general modeling process of the part family model, and according to the characteristics of its own configuration function, introduces two parts family information extraction 
methods based on multiple configurations and design tables. 5) Taking UG as an example, it focuses on related technologies related to two-dimensional engineering drawing services. Since PDF has the advantages of fast signing speed and high signing efficiency, the import of engineering drawings will try to generate PDF files. The method of converting two-dimensional engineering drawings to PDF further adopts the engineering drawing signature method and signature process of PDM.

Yujie Sun, https://orcid.org/0000-0001-7747-2872

\section{REFERENCES}

[1] Gupta, A.; Kumar, R.; Arora, H.: C-CADZ: computational intelligence system for coronary artery disease detection using Z-Alizadeh Sani dataset, Applied Intelligence, 5(5) , 2021, 113. https://doi.org/10.1007/s10489-021-02467-3

[2] Arvor, D.; Betbeder, J.; Daher, F.: Towards user-adaptive remote sensing: Knowledge-driven automatic classification of Sentinel-2 time series, Remote Sensing of Environment, 264(17), 2021, 112615. https://doi.org/10.1016/j.rse.2021.112615

[3] Tran, T.-A.; Lobov, A.; Kaasa, T.-H.: CAD integrated automatic recognition of weld paths, The International Journal of Advanced Manufacturing Technology, 115, 2021, 7-8. https://doi.org/10.1007/s00170-021-07186-0

[4] Khan, M.-T.-H.; Demoly, F.; Kim, K.-Y.: Constructing assembly design model capable of capturing and sharing semantic dynamic motion information in heterogeneous CAD systems. The International Journal of Advanced Manufacturing Technology, 111(3), 2020, 945-961. https://doi.org/10.1007/s00170-020-06046-7

[5] Barladian, B.-K.; Voloboy, A.-G.; Galaktionov, V.-A.; Shapiro, L.-Z.: Integration of realistic computer graphics into computer-aided design and product lifecycle management systems. Programming and Computer Software, 44(4), 2018, 225-232. https://doi.org/10.1134/S0361768818040047

[6] Relvas, C.; Ramos, A.: New methodology for product development process using structured tools, Proceedings of the Institution of Mechanical Engineers, Part B: Journal of Engineering Manufacture, 235(3), 2021, 378-393. https://doi.org/10.1177/0954405420971228

[7] Wang, X.; Bi, Z.: New CAD/CAM course framework in digital manufacturing, Computer Applications in Engineering Education, 27(1), 2019, 128-144. https://doi.org/10.1002/cae.22063

[8] Cai, X.-T.; Wang, S.: Design gene based secure mechanism for collaborative product development, Advanced Engineering Informatics, 47(20), 2021, 10122. https://doi.org/10.1016/j.aei.2020.101228

[9] Krystek, J.; Alszer, S.; Bysko, S.: Computer aided design of sequencing problem-the concept of Paint Shop 4.0. Mechanik, 91(7), 2018, 529-531. https://orcid.org/0000-0003-2015-3453

[10] Mokni, R.; Gargouri, N.; Damak, A.: An automatic Computer-Aided Diagnosis system based on the Multimodal fusion of Breast Cancer (MF-CAD), Biomedical Signal Processing and Control, 69(11), 2021, 102914. https://doi.org/10.1016/j.bspc.2021.102914 